J. Med. Microbiol. - Vol. 46 (1997), 887-892

(C) 1997 The Pathological Society of Great Britain and Ireland

\title{
CORRESPONDENCE
}

\section{A novel mechanism of antibiotic resistance in plague?}

The specific therapy for plague, as suggested by the WHO Expert Committee on Plague (1970), involves the use of antibiotics such as tetracycline, streptomycin, and chloramphenicol. It is accepted that Yersinia pestis, the causative agent of plague, in contrast to the majority of other pathogenic bacteria, lacks antibiotic resistance in vitro and in vivo. The absence of natural drug-resistant strains may be explained by the rarity of cases of human plague at the present time and the acute character of the disease, and the flea transmission mechanism of the infection which limits contact between $Y$. pestis and natural reservoirs of $\mathrm{R}$ factors.

However, it has been shown that the treatment of plague caused by non-capsulate natural or experimental $Y$. pestis strains was less effective when antibiotics such as doxycycline, ampicillin and cefoperazone were used. The non-capsulate strains were also resistant to tetracycline, $\beta$-lactam agents and quinolones when inside macrophages cultivated in vitro [1-3]. In some cases, the loss of ability to produce capsules did not lead to an increase of LD50 absolute values in the infected mice and guinea-pigs [4]. Microbial pathogenesis is complex and mutifactorial, and several virulence factors may act individually or in concert to produce infection. Removal of any one of these components may or may not render the organism avirulent. Moreover, non-capsulate strains may be isolated from fatal cases of human [5] and rodent plague. Interestingly, when the $Y$. pestis cultures were grown on nutrient media all the non-capsulate strains were as sensitive to antibiotics as capsulate wild-type ones [1-3]. No attempts have been made to explain this phenomenon. Here we propose factors that might determine this novel mechanism of antibiotic resistance.

The pathogenicity of $Y$. pestis is related to its intracellular survival and multiplication in macrophages. Released bacteria are resistant to capture by neutrophils, inside which $Y$. pestis cells can be killed, but those phagocytosed by mononuclear phagocytes begin a new cycle of intracellular propagation [6]. It is also known that some antibiotics are unable to enter macrophages and their phagolysosomes in active form. On the other hand, the extracellular life cycle of $Y$. pestis cell may be too short for a bactericidal affect, while the other, bacteriostatic antibiotic action can only prevent the growth of $Y$. pestis outside macrophage. Phagocytosed non-capsulate bacteria are protected from contact with the antibiotic and are able to multiply. It seems likely that the capsule of $Y$. pestis may influence macrophage-membrane permeability for a range of antibiotics.

Capsular antigen "fraction I" (FI) forming the $Y$. pestis capsule is specific to $Y$. pestis and induces a pronounced antiphagocytic effect in a non-immune host. [6] It has been suggested that the FI antigen, a mature polypeptide, inhibits the activity of phagocytic cells by forming large aqueous pores in the membrane of these target cells [7]. Also, it has been shown that channel formation leads to the leakage of $\mathrm{K}^{+}, O_{-}^{-}$ nitrophenylgalactoside, and other low-mol.wt molecules through cell membranes after channel-forming toxin treatment [8]. All things considered, we can speculate a priori that, in vivo, the FI antigen-induced aqueous pores may be the main route of antibiotic penetration into macrophages and phagolysosomes resulting in antibiotic susceptibility in $Y$. pestis wildtype strains. Schematic representation of events leading to the killing of the intracellular $\mathrm{FI}^{+}$bacteria may be as shown in the Figure. Step 1 corresponds to capsular antigen secretion into the media surrounding $Y$. pestis [9], the extramacrophage and intraphagolysosomal spaces. The dissolved FI antigen makes contact with the extracytoplasmic surface of the macrophage and phagolysosome membranes (step 2), and in inserted into the lipid bilayer plasma membranes forming the aqueous channels (step 3), facilitating the penentration of more FI into the cytoplasm and FI-pore-forming insertion into the Golgi-apparatus and endoplasmic-reticulum membranes (step 4). The damaged macrophage is unable to prevent antibiotic contact with the capsule-producing bacteria (step 5).

If this hypothesis is correct, the ability of the wildtype $Y$. pestis to enter the macrophage seems to be something of a paradox. It is hoped that further work in this area will clarify the molecular mechanisms by which $Y$. pestis capsular antigen affects antibiotic susceptibility of this microbe.

A.P. Anisimov and I.A. Dyatlov* Laboratory of Applied Genetics and ${ }^{*}$ Department for Specialist Training, Russian Research Anti-Plague Institute "Microbe", Universitetskaya Street 46, 410005, Saratov, Russia.

\section{References}

1. Samokhodkina ED, Ryzhko IV, Shcherbaniuk AI, Kasatkina IV, Tsuraeva RI, Zhigalova TA [Doxycycline in the prevention of experimental plague induced by plague microbe variants.] Antibiot Khimioter 1992; 37: 26-28. 
A
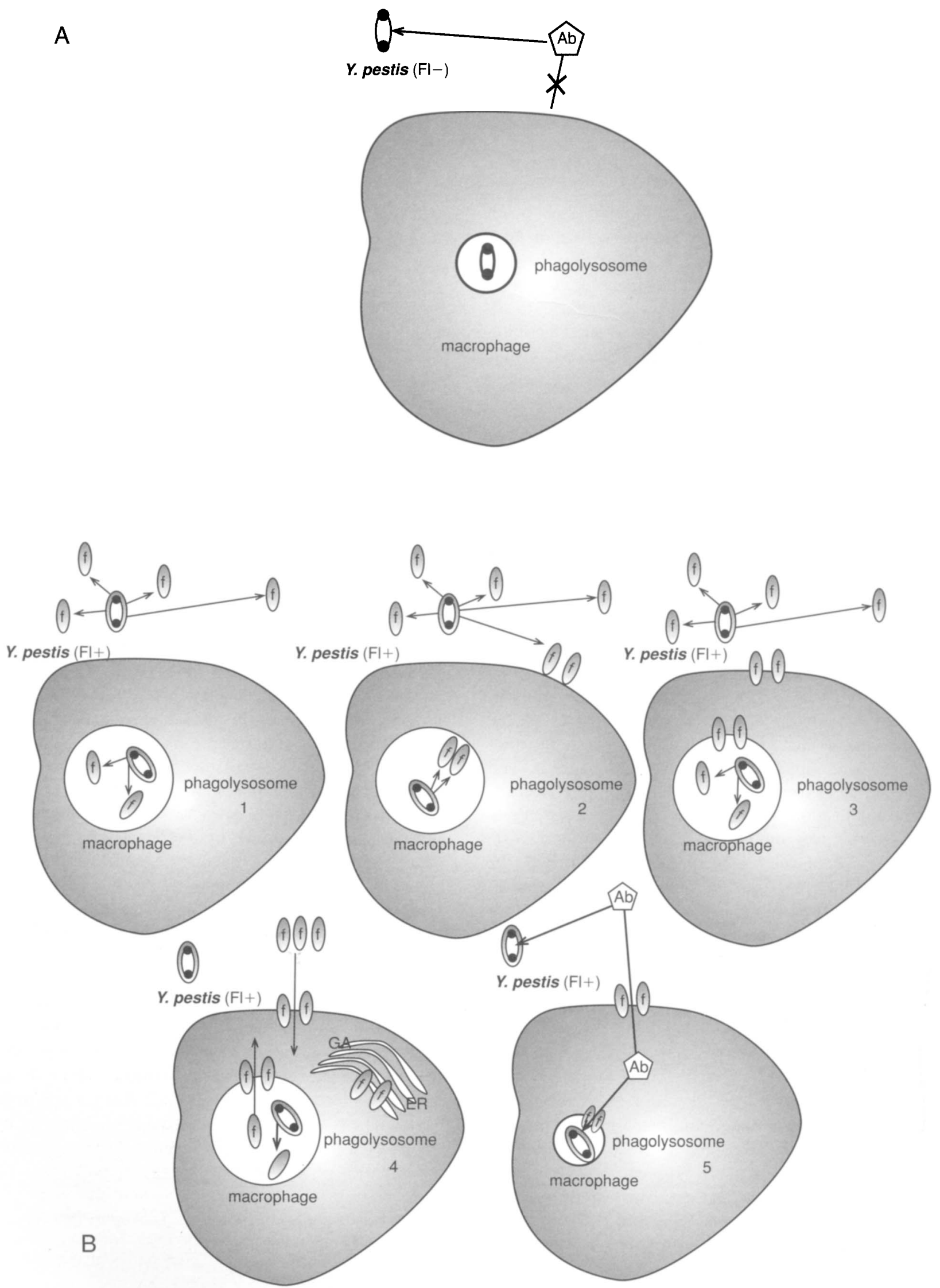

Fig. 1. Possible effect on non-capsulate $\left(\mathrm{FI}^{-}\right)$and wild-type $\left(\mathrm{FI}^{+}\right)$Y. pestis cells when an antibiotic $(\mathrm{Ab})$ is added to a cell culture of macrophages mixed with the bacteria. The antibiotic expresses its antimicrobiol effect $(\rightarrow)$ when in contact with extracellular microbes $(A, B)$. It is unable to affect $(-)$ the intracellular capsule-deficient microbial cells (A). The wild-type bacteria secrete the "fraction I" capsular antigen into the surrounding milieu [9]. The dissolved antigen FI forms the aqueous channels in macrophage membranes (1), enabling contact between the antibiotic and the capsule-producing microbes (B). 
2. Samokhodkina ED, Ryzhko IV, Tsuraeva RI, Pasiukov VV. [Beta-lactam antibiotics (ampicillin, cefotaxime) in prevention of experimental plague in albino mice, caused by nonfractioned strains of the pathogen.] Antibiot Khimioter 1994; 39: $20-23$.

3. Pasuikov VV, Ryzhko IV, Tsuraeva RI, Samokhodkina ED, Roshchina NV. [Cefoperazone in the prevention and treatment of experimental plague caused by typical and fraction-free pathogen strains in white mice.] Antibiot Khimioter 1994; 39: 37--40.

4. Drozdov IG, Anisimov AP, Samoilova SV et al. Virulent noncapsulate Yersinia pestis variants constructed by insertion mutagenesis. J Med Microbiol 1995; 42: 264-268.

5. Winter CC, Cherry WB, Moody MD. An unusual strain of Pasteurella pestis isolated from a fatal human case of plague.
Bull WHO 1960; 23: 408-409.

6. Cavanaugh DC, Randall R. The role of multiplication of Pasteurella pestis in mononuclear phagocytes in the pathogenesis of flea-borne plague. $J$ Immunol 1959; 83: 348-363.

7. Rodrigues CG, Carneiro CM, Barbosa CT, Nogueira RA. Antigen F1 from Yersinia pestis forms aqueous channels in lipid bilayer membranes. Braz J Med Biol Res 1992; 25: 75-79.

8. Kagan BL, Selsted ME, Ganz T, Lehrer RI. Antimicrobial defensin peptides form voltage-dependent ion-permeable channels in planar lipid bilayer membranes. Proc Natl Acad Sci USA 1990; 87: 210-214.

9. Chen TH, Elberg SS. Scanning electron microscopic study of virulent Yersinia pestis and Yersinia pseudotuberculosis type I. Infect Immun 1977; 15: 972-977.

\section{Application of pulsed-field gel electrophoresis in an outbreak of infection due to Klebsiella oxytoca}

In recent years several cases of nosocomial septicaemia caused by Klebsiella oxytoca have been reported [1-4]. $K$. oxytoca strains are usually differentiated on the basis of capsular serotype or biotype. Pulsed field gel electrophoresis (PFGE) has been used to study nosocomial septicaemia caused by $K$. pneumoniae [5] but not for $K$. oxytoca. We report here an outbreak of nosocomial infection of $K$. oxytoca in leukaemia patients. In one week, $K$. oxytoca was isolated from the blood cultures (Kox 1-6) of six immunosuppressed (neutropenic) patients, all of whom were in the same ward and all had a Hickman-type central venous catheter in situ which was anticoagulated with a heparin solution from a common vial. It was important to demonstrate that this was a true outbreak following contamination with a single strain.

$K$. oxytoca isolates were identified and their antimicrobial susceptibility was tested by MicroScan NegCombo 6I panel (Baxter Diagnostics Inc.). Genomic DNA was prepared in low-melting-point agarose plugs. Bacteria, freshly grown on MüellerHinton agar, were suspended in TE buffer $(10 \mathrm{mM}$ Tris, $\mathrm{pH} 8,0.1 \mathrm{mM}$ EDTA) to an $\mathrm{OD}_{650}$ of 1.250 representing $\left(1 \times 10^{9}\right)-\left(5 \times 10^{9}\right)$ bacteria $/ \mathrm{ml}$. Bacterial suspensions were mixed with an equal volume of agar $1 \%$ in TE buffer. The mixture was incubated in $3 \mathrm{ml}$ of lysis buffer (TE buffer, lauryl-sarcosine 1\%, pronase $1 \mathrm{mg} / \mathrm{ml}$ ) for $48 \mathrm{~h}$ at $37^{\circ} \mathrm{C}$. Agarose plugs were washed once for $1 \mathrm{~h}$ at $37^{\circ} \mathrm{C}$ and once for $1 \mathrm{~h}$ at room temperature in phenylmethylsulphonyl fluoride solution and then three times in TE buffer at room temperature. DNA in the plugs was digested overnight at $37^{\circ} \mathrm{C}$ with $40 \mathrm{U}$ of $X b a \mathrm{I}$ in the manufacturer's recommended buffer. DNA fragments were separated by electrophoresis in an agarose $1 \%$ gel prepared and run in $0.5 \times$ Tris-borate-EDTA (TBE) buffer in a contour-clamped homogeneous electric field machine (CHEF-DR2; Bio-Rad). The pulse times were $20 \mathrm{~s}$ for $24 \mathrm{~h}$ at $180 \mathrm{~V}$. The gels were then stained with ethidium bromide and photographed under UV light.

The Hickman-type central venous catheter was the putative clinical focus of infection because the cath- eter site was erythematous in all cases. Cultures obtained from the Hickman-type catheters (isolates Kox 7-12) from the six patients yielded $K$. oxytoca with antibiograms similar to those of isolates (Kox 1 6) obtained from blood cultures. In the course of the investigation, our attention was directed to the heparin solution from which an isolate of $K$. oxytoca with the same antibiogram was isolated (Kox 14).

During the week of the outbreak, all leukaemia patients had been moved to the Urology Department where the majority of infections took place. This led us to compare sporadic isolates of $K$. oxytoca from patients admitted to the Urology and Haematology Departments previously or during the outbreak (Kox 13 and Kox 15-20).

To determine the extent and source of the outbreak, DNA from all $K$. oxytoca isolates was digested by $X b a \mathrm{I}$ and separated by electrophoresis. As shown in Fig. 1a, the Kox 1-6 isolates from the leukaemia patients (Lanes 1-6) showed the same electrophoretic patterns of 17 identical fragments. Isolates Kox 7-12 from Hickman-type catheters (Fig. 1b, lanes 7-12) and Kox 14 from heparin solution (lane 14) exhibited banding patterns identical to the blood culture isolates. Strain Kox 13 isolated during the outbreak from the urology patient showed a different antibiogram and fingerprint (Fig. 1b, lane 13). The DNA fingerprints of the sporadic isolates Kox 15-20 obtained before the outbreak (Fig. 1c, lanes 15 to 20 ) were all different and distinct from the former patterns (Fig. 1a, lanes 1-6), except isolate Kox 15 from a blood culture of a urology patient 4 days before the outbreak. This isolate had the same restriction pattern and antibiogram as the outbreak strain (Fig. 1c, lane 15).

The striking homogeneity of DNA fingerprints after $X b a \mathrm{I}$ analysis of isolates Kox 1-12, Kox 14 and Kox 15 compared with the heterogeneity shown by isolates Kox 16-20, suggest that this was a true epidemic as a result of the spread of a single $K$. oxytoca strain, from a single source, the heparin solution. Furthermore, because all the leukaemia patients were moved to the Urology Department, the urology patient who had an 
$\begin{array}{lllllll}A & 1 & 2 & 3 & 4 & 5 & 6\end{array}$

a

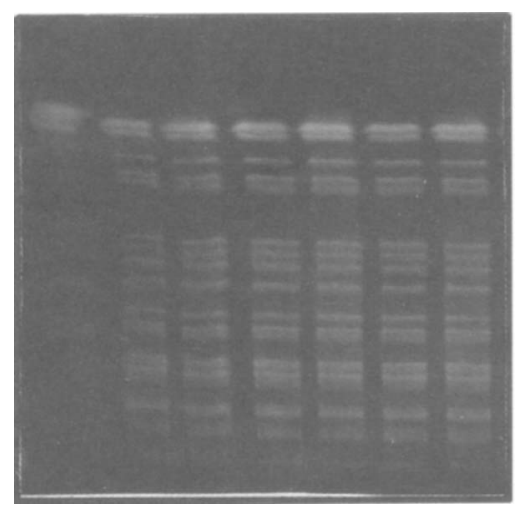

$\begin{array}{lllllllll}\text { A } & \text { B } & 15 & 16 & 17 & 18 & 19 & 20 & \text { C }\end{array}$

C

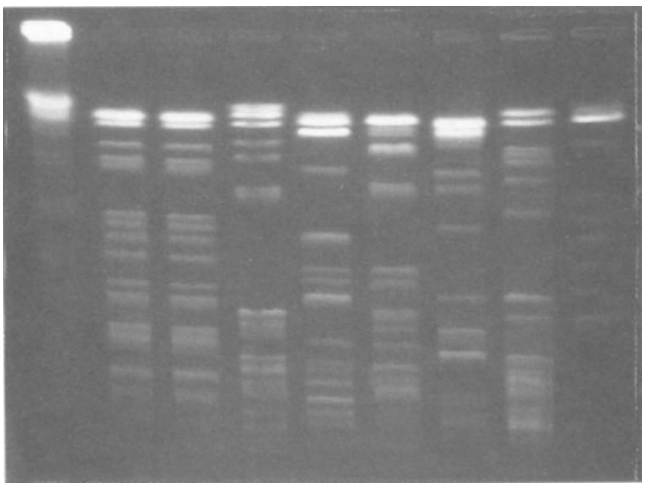

identical K. oxytoca isolate Kox 15 may have been the original source of contamination of the heparin solution. On the other hand, this patient may have been infected by Kox 15 by manipulations by attendant staff though the timing makes this less likely.

Carmen M. Toldos, Gines Ortiz, Margarita Cámara and Manuel Segovia Servicio de Microbiobiologia, Hospital General Universitario, Avda. Intendente Jorge Palacios, I30003 Murcia, Spain.

\section{Acknowledgments}

We thank Prof. C. A. Hart for his critical comments.

\section{$\begin{array}{llllllllll}A & 7 & 8 & 9 & 10 & 11 & 12 & 13 & 14 & B\end{array}$}

b

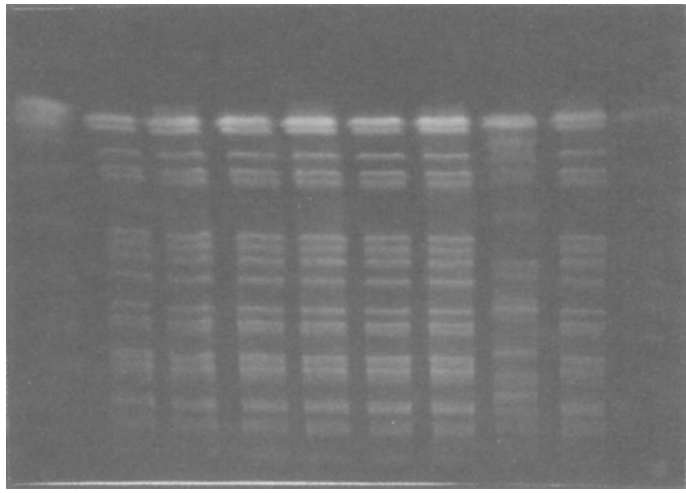

Fig. 1. PFGE of total DNA digested by XbaI of $K$. oxytoca isolates. (a) Lane $\mathbf{A}$, lambda concatamer; 1-6, blood culture isolates from the six haematology patients. (Kox 1-6). (b) Lane A, lambda concatamer; 7-12, isolates from Hickman-type central venous catheter in the six haematology patients (Kox 7-12); 13, Kox 13 (from a urology patient); 14, Kox 14 (from heparin solution); B, K. pneumoniae cut by Xbal. (c) Lane A, lambda concatamer; B, Kox 1 from outbreak; 15, Kox 15 (from a urology patient 4 days before the outbreak, similar antibiogram); 16-20, sporadic isolates (Kox 1620) from urology and haematology patients before the outbreak; C, $K$. pneumoniae cut by XbaI.

\section{References}

1. Fryklund B, Tullus K, Burman LG. Epidemiology and attack index of gram-negative bacteria causing invasive infection in three special-care neonatal units and risk factors for infection. Infection 1995; 23: 76-80.

2. Haertl R, Bandlow G. Use of small fragment restriction endonuclease analysis (SF-REA) for epidemiological fingerprinting of Klebsiella oxytoca. Int $J$ Med Microbiol Virol Parasitol Infect Dis 1994; 280: 312-318.

3. Korvick JA, Bryan CS, Farber B et al. Prospective observational study of Klebsiella bacteremia in 230 patients: outcome for antibiotic combinations versus monotherapy. Antimicrob Agents Chemother 1992; 36: 2639-2644.

4. Ranjsö U, Good Z, Jalakas K et al. An outbreak of Klebsiella oxytoca septicemias associated with the use of invasive blood pressure monitoring equipment. Acta Anaesthesiol Scand 1992; 36: $289-291$

5. Gouby A, Neuwirth C, Bourg G et al. Epidemiological study by pulsed-field gel electrophoresis of an outbreak of extendedspectrum $\beta$-lactamase-producing Klebsiella pneumoniae in a geriatric hospital. J Clin Microbiol 1994; 32: 301-305.

\section{Diagnosis of ventilater-associated pneumonia}

We read with interest the article by Humphreys et al. ( $J$ Med Microbiol 1996; 45: 226-231) investigating the use of Foley catheters in the diagnosis of ventilatorassociated pneumonia (VAP). We agree that the clinical diagnosis of VAP is difficult and the "gold standard" requires extensive histopathological examination of the lungs, only possible at post-mortem examination. Ventilated patients frequently develop pyrexia and radiological infiltrates on chest X-ray. Many such episodes are treated with empirical antibiotics and "diagnosed" retrospectively as VAP. This approach has been used in published diagnostic studies [1]. It is more accurate to regard such patients as clinically suspect VAP, in the absence of confirmation. However, concurrent bacteraemia with an identical organism in lower respiratory tract secretions and blood cultures, in the absence of an alternative source of bacteraemia, has been regarded as diagnostic of VAP [2]. In the study by 
Humphreys et al. no data on the confirmation of VAP diagnosis in suspected cases were given. Their clinical diagnostic criteria did not necessitate the presence of chest X-ray infiltrates or worsening gas exchange. The bacteriological thresholds are confusing as both positive and equivocal culture results are defined by the presence of pure growths at $10^{4} \mathrm{cfu} / \mathrm{ml}$. Also, the presence of polymorphs is a sensitive but non-specific finding in VAP [3] and should not render a pure growth at $10^{4} \mathrm{cfu} / \mathrm{ml}$ as being equivocal.

We are investigating the diagnostic utility of nondirected bronchial lavages (NBL) in VAP, using simple tracheal suction catheters and quantitative cultures. Quantitation is with filter paper dipstrips (Bacteruritest strips) on twice weekly NBL specimens. All episodes of suspected VAP are investigated with sequential NBL, bronochoscopic bronchoalveolar lavage and protected specimen bronchial brush to compare directly the operating characteristics of each technique. Interim results on 130 adults, including 32 episodes of suspected VAP, have revealed that NBL has similar diagnostic reliability to invasive bronchoscopic techniques and can be used as a primary diagnostic method. Bronchoscopy should be reserved for those patients who fail to improve on appropriate antibiotics, have sterile NBL cultures in the absence of prior antimicrobial treatment or acute respiratory distress syndrome (ARDS). Moreover, a single threshold of bacterial load to distinguish colonisation or contamination from VAP is inappropriate and should vary depending on the length of ventilation and whether concurrent antibiotics are being administered.

P.G. Flanagan and R.A. Barnes Department of Medical Microbiology and Public Health Laboratory, University of Wales College of Medicine, Heath Park, Cardiff CF4 4XN.

\section{References}

1. A'Court CHD, Garrard CS, Crook D et al. Microbiological lung surveillance in mechanically ventilated patients using nondirected bronchial lavage and quantitative culture. $Q \mathrm{~J} \mathrm{Med}$ 1993; 86: 635-648.

2. Pingleton SK, Fagon J-Y, Leeper KV. Patient selection for clinical investigation of ventilator-associated pneumonia. Chest 1992; 102: $553 \mathrm{~S}-556 \mathrm{~S}$

3. Pugin J, Auckenthaler R, Mili N et al. Diagnosis of ventilatorassociated pneumonia by bacteriologic analysis of bronchoscopic and non-bronchoscopic blind bronchoalveolar lavage fluid. $\mathrm{Am}$ Rev Respir Dis 1991; 143: 1121-1129.

\section{Reply: Diagnosis of ventilator-associated pneumonia}

We are grateful to Drs Flanagan and Barnes for their comments on our recent study [1]. Confirming a diagnosis of ventilator-associated pneumonia remains problematic in the majority of cases in the absence of tissue for histological and microbiological analysis. We compared two different techniques to obtain specimens for microbiological processing in a routine laboratory but did not seek to confirm a diagnosis by histology or at post-mortem examination. We did, however, include changes in the chest X-ray appearance and a deterioration in gas exchange (see patients and procedure) as indicators of infection necessitating broncho-alveolar or catheter lavage.

The criteria used to indicate the presence of infection, i.e., number and type of cells seen on microscopy and the numbers or organisms cultured, vary from study to study but it is now generally agreed that quantitation is important in distinguishing infection from contamination [2]. In a recent study which compared the results from bronchoscopy specimens taken just after death with histological assessment and quantitative lung tissue culture at post-mortem examination, a threshold for diagnosis of $>10^{4} \mathrm{cfu} / \mathrm{ml}$ resulted in diagnostic sensitivity and specificity of $91 \%$ and $78 \%$, respectively [3]. Our criteria were based on the results of similar previous studies but were adapted for use in a routine laboratory and allowed for important clinical variables such as recent or concurrent antimicrobial therapy resulting in smaller numbers or organisms being isolated. In routine clinical practice some degree of judgement is desirable when deciding whether or not to start antimicrobial agents or to change to second or third line agents. Recent standardised procedures to obtain and process respiratory specimens from patients with ventilator-associated pneumonia recommend the use of a Wright-Giemsa stain to obtain a differential cell count, including the number of polymorphonuclear cells present [4, 5]. The use of this stain is not routine in our respiratory laboratory but perhaps this should be considered when attempting to confirm a diagnosis of pneumonia in this group of acutely ill patients.

The preliminary results of Drs Flanagan and Barnes comparing quantitative non-directed bronchial lavage (NBL) with quantitation of simple tracheal suction catheter specimens confirm the usefulness of NBL. We agree with their clinical indications for bronchoscopy but would also include the need to exclude malignancy in patients with an abnormal chest X-ray and the need for suction in a patient with possible infection and a collapsed lung segment. Finally, we agree that a single threshold of bacterial load is inappropriate. Ultimately, improvements in the diagnosis and management of ventilator-associated pneumonia are likely to be assisted by greater dialogue between intensive care physicians and microbiologists.

H. Humphreys, Robert Winter* and M. Baker Division of Microbiology and Infectious Diseases, Public Health 
Laboratory and *Adult Intensive Care Unit, University Hospital, Queens Medical Centre, Nottingham NG7 2UH, UK.

\section{References}

1. Humphreys H, Winter R, Baker M, Smith C. Comparison of bronchoalveolar lavage and catheter lavage to confirm ventilatorassociated lower respiratory tract infection. $J$ Med Microbiol 1996; 45: 226-231
2. Baselski VS, Wunderink RG. Bronchoscopic diagnosis of pneumonia. Clin Microbiol Rev 1994; 7: 533-558.

3. Chastre J, Fagon J-Y, Bornet-Lesco $\mathrm{M}$ et al. Evaluation of bronchoscopic techniques for the diagnosis of nosocomial pneumonia. Am J Respir Crit Care Med 1995; 152: 231-240.

4. Meduri G, Chastre J. The standardization of bronchoscopic techniques of ventilator-associated pneumonia. Chest 1992; 102 $557 \mathrm{~S}-564 \mathrm{~S}$.

5. Baselski VS, El-Torky M, Coalson JJ, Griffin JP. The standardization of criteria for processing and interpreting laboratory specimens in patients with suspected ventilatorassociated pneumonia. Chest 1992; 102: 571S-579S. 